\title{
An Unusual but Important Cause of Hyperandrogenism in Women
}

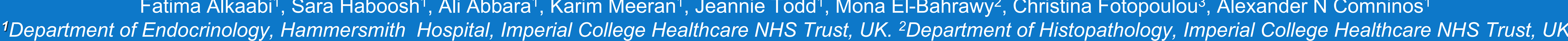 ${ }^{3}$ Department of Gynaecology, Queen Charlotte's \& Chelsea Hospital, Imperial College Healthcare NHS Trust, UK}

\section{Introduction}

New onset hyperandrogenism in a woman is a rare but important presentation to the endocrine clinic; due to the marked and often alarming effects on the patient, and the variety of causes each with a different management approach.

Causes of hyperandorgenism in females includes polycystic ovarian syndrome, congenita adrenal hyperplasia, Cushing's syndrome, ovarian hyperthecosis, and androgen-secreting tumours.

\section{Case Description}

A 61 year-old woman presented with a two year history of worsening facial hirsutism and frontal balding (Figure 1).

- She did not report any deepening of voice, acne, change in body habitus or symptoms of Cushing's syndrome.

Menarche was at age 14y, with regular menses until a hysterectomy (with ovarian preservation) for menorrhagia aged $29 y$.

She had a past medical history of T2DM and gastric bypass surgery.

She was not on any androgenic medication.

Examination revealed clinical hyperandrogenism with hirsutism (FG score 20), androgenic alopecia, and coarse facial features but no cliteromegaly.

There were no clinical features of Cushing's syndrome or other endocrine disease. Secondary sexual characteristics and remaining examination were otherwise normal.

Blood tests revealed marked biochemical hyperandrogenism:

Testosterone $\quad 5.7 \mathrm{nmol} / \mathrm{l}(0-2)$

Androstenedione $\quad 1.6 \mathrm{nmol} / \mathrm{l}(0-9)$

DHEAS

$0.8 \mathrm{umol} / \mathrm{l}(0.4-4.7)$

Oestradiol $\quad<70 \mathrm{pmol} / \mathrm{l}$

LH 22.1iu/l (postmenopausal)

FSH 44.3iu/l (postmenopausal)

SHBG $\quad 27 \mathrm{nmol} / \mathrm{l}(30-100)$

$\mathrm{HbA1c} \quad 63 \mathrm{mmol} / \mathrm{mol}(20-41)$

Prolactin, AFP, hCG, IGF1 were within the reference range

- LDDST resulted in suppressed cortisol and DHEAS but no suppression of testosterone suggesting an ovarian source.

- MRI adrenals and US pelvis were unremarkable with normal-size ovaries (4.1 and $3.1 \mathrm{ml}$ ).

\section{Clinical Course}

- Based on the likely ovarian androgen source with unremarkable US ovaries, she was initially diagnosed with ovarian hyperthecosis and commenced on monthly GnRHanalogue therapy.

However, on GnRH-analogue therapy, her testosterone levels were still consistently raised (2.3-3.8nmol/l) albeit slightly lower.

- Given the rapidity of the hirsutism, the normal-sized ovaries on US, and the failure to suppress adequately with $\mathrm{GnRH}$-analogues, the diagnosis was re-visited.

Subsequent MRI pelvis offered superior resolution for ovarian pathology (over previous normal US) and revealed a $1 \mathrm{~cm}$ left ovarian mass (Figure 2 ).

- She subsequently underwent laparoscopic bilateral salpingo-oophorectomy and the histopathology identified an ovarian Leydig cell tumour (Figures 3 and 4).

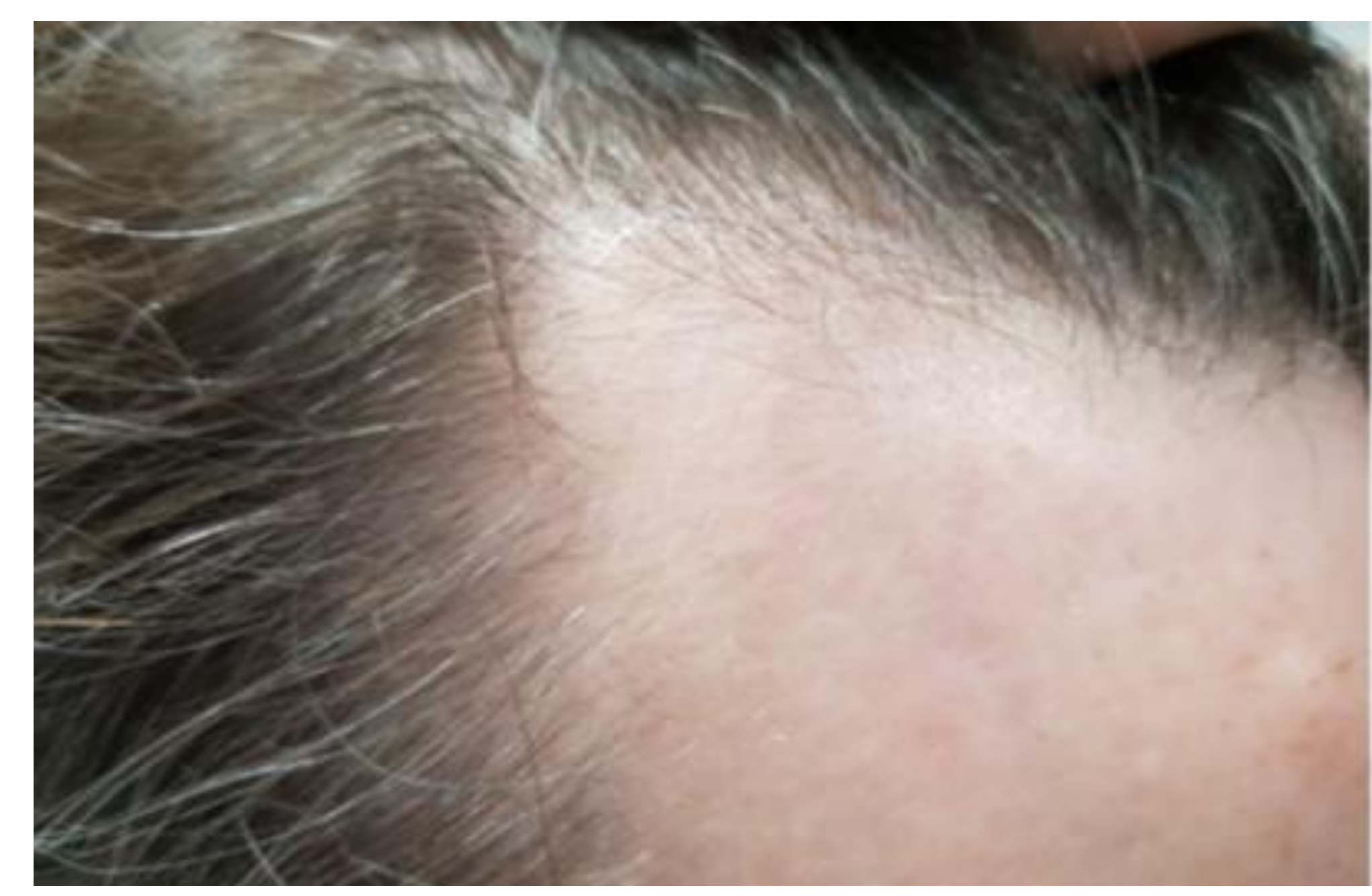

Figure 1: Frontal balding (with permission from patient)

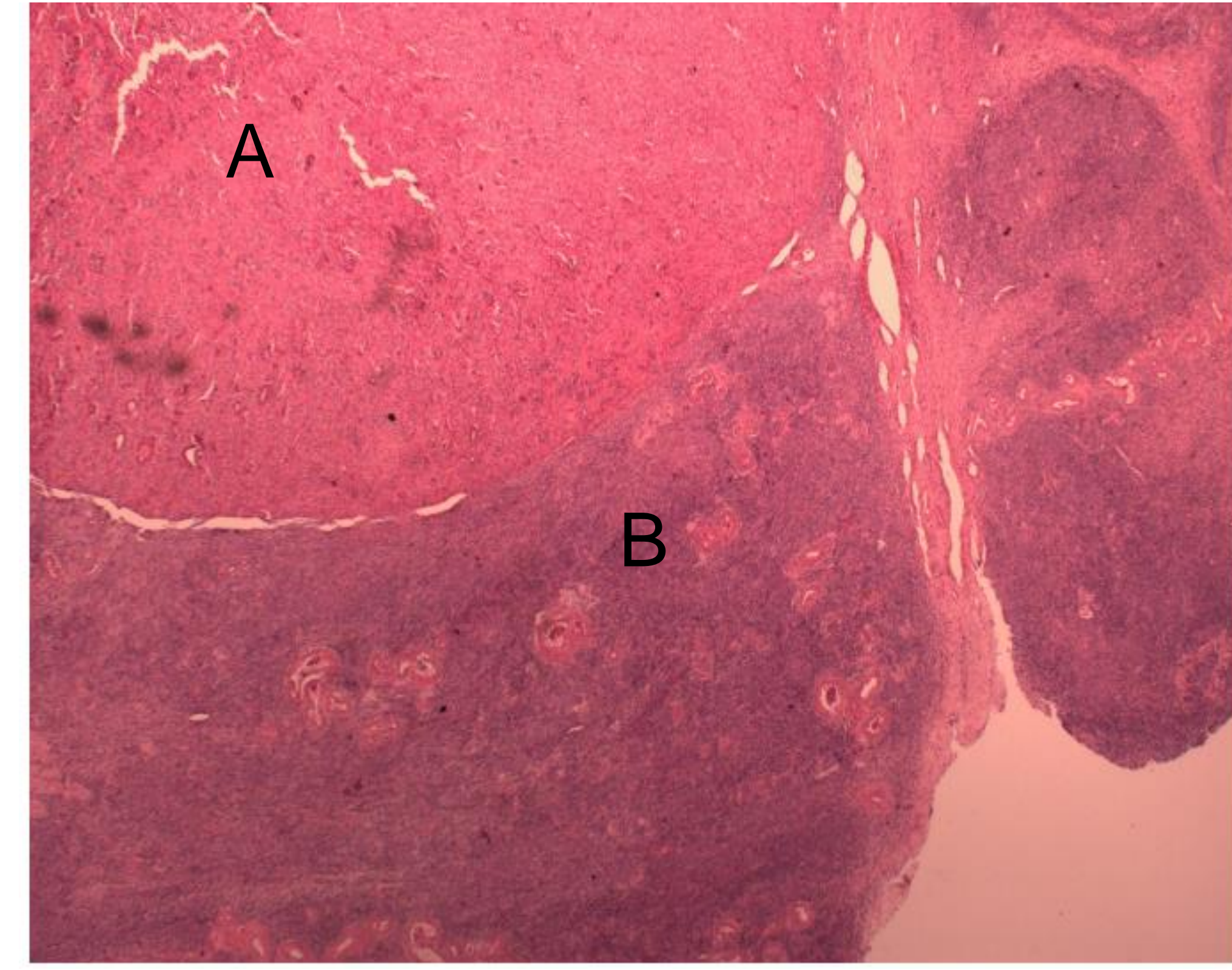

Figure 3: Well circumscribed Leydig tumour (A) with adjacent ovarian tissue (B)(x20 magnification)
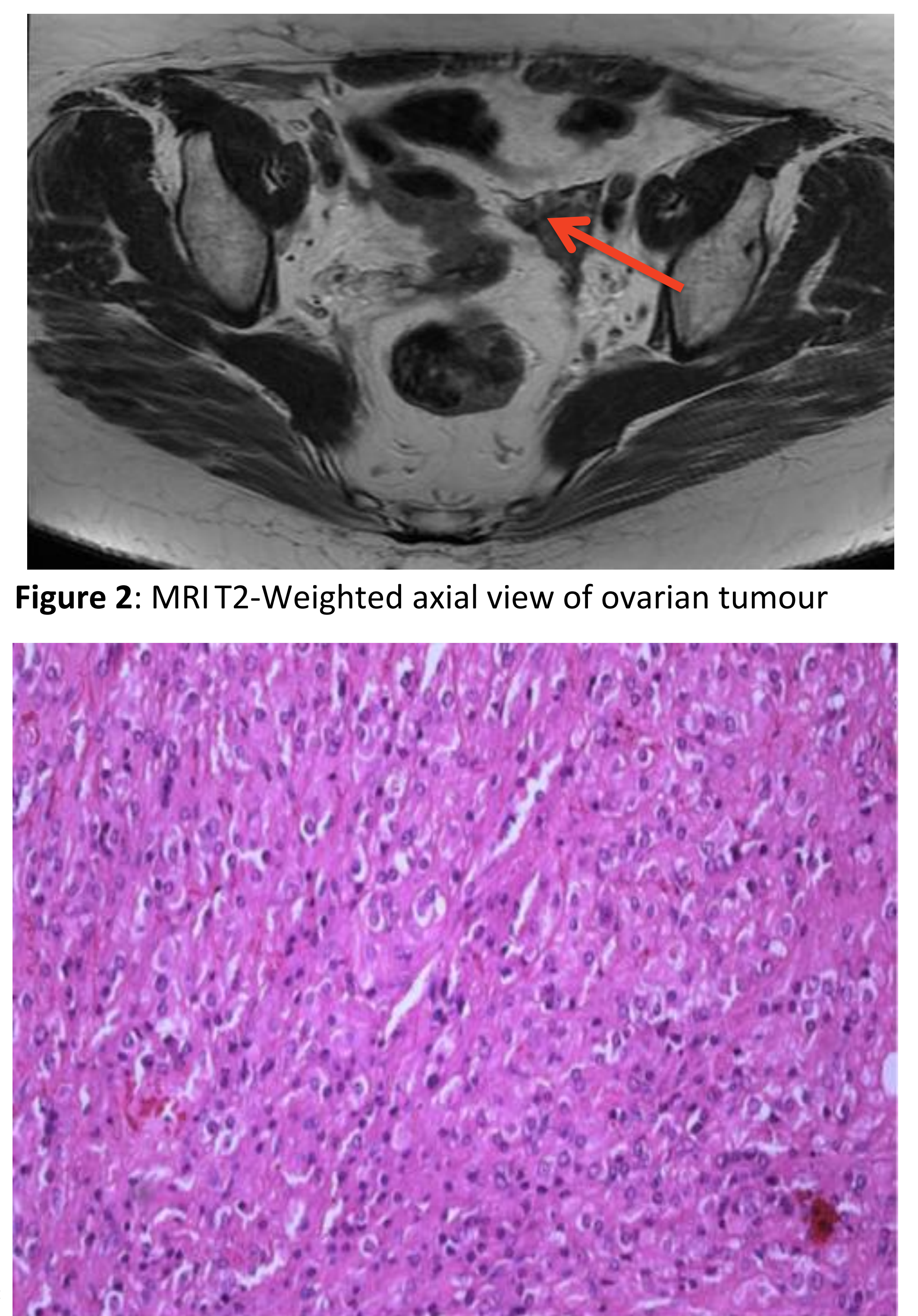

Figure 4: Leydig tumour at higher magnification $(\times 200)$ polygonal cells with ample brightly eosinophilic cytoplasm and regular round nuclei.

\section{Case Outcome}

Four months post-surgery : Testosterone $0.5 \mathrm{nmol} / \mathrm{I}, \mathrm{LH} 2.9 \mathrm{iu} / \mathrm{I}, \mathrm{FSH} 10.6 \mathrm{iu} / \mathrm{I}$.

Six months post-surgery her testosterone remains low off LHRH-analogues, with gradual improvement of her hirsutism and stabilisation of her alopecia.

\section{Discussion}

The presence of androgen-secreting tumors should be considered carefully when the serum testosterone level is $>5.2 \mathrm{nmol} / \mathrm{l}$ and/or when progressive hyperandrogenism appear abruptly, particularly in postmenopausal women (1-3)

In addition to the noticeable virilising physical effects of elevated testosterone, hyperandrogenism is also associated with hypercholesterolemia, insulin resistance, hypertension, and cardiac disease as well as the oncological consequences of any tumour. Therefore identification and elimination the source of testosterone is essential (4).

In premenopausal women, the most common cause of hyperandrogenism is polycystic ovary syndrome. In contrast, when hyperandrogenism develops in postmenopausal women, it is often associated with other causes, such as ovarian hyperthecosis or an androgen-secreting tumour.

Ovarian hyperthecosis is a disorder characterised by severe hyperandrogenism and insulin resistance. Women usually present with slowly progressive acne and hirsutism (and they are likely to be virilised) (5-7). US usually reveals bilaterally enlarged ovaries (unlike in this patient, which contributed to us questioning the diagnosis).

- Androgen-secreting tumours usually present with rapidly progressive hyperandrogenism resulting in virilisation. These tumors originate from the adrenal glands or ovaries (2).

- Androgen-secreting adrenal tumours are very uncommon, large, aggressive and usually associated with high cortisol levels. However purely androgen-secreting adrenal tumors also exist (2).

- Androgen-secreting ovarian tumours are also rare, representing less than $0.2 \%$ of all causes of hyperandrogenism and fewer than $1 \%$ of all ovarian tumors (2).

- Sertoli-Leydig cell tumors constitute fewer than $0.5 \%$ of ovarian tumors and may be benign or malignant. Crucially they may be small enough to avoid detection even with careful radiological studies leading to bilateral oophorectomy as both a diagnostic and therapeutic approach $(2,5,8)$. This mirrors our case where the tumour was not seen on US but was seen on MRI. In some cases, ovarian sampling can be used to definitively identify the source of the androgens (especially in pre-menopausal women seeking future fertility where unilateral oophorectomy would be preferred).

- Our patient had an elevated testosterone, normal DEHAS and non-suppressible testosterone on LDDST. Her Ultrasound pelvis and MRI adrenals were both unremarkable. She was initially diagnosed with ovarian hyperthecosis but failure of the $\mathrm{GnRH}$ therapy to suppress her testosterone into the normal range and her normal-sized ovaries, led us to re-visit her diagnosis. In this case, MRI offered superior resolution of the ovaries and detected a small ovarian tumour.

- Oophorectomy is the treatment of choice (in good surgical candidates) for both ovarian hyperthecosis (bilateral) and ovarian tumors (uni or bilateral) $(9,10)$.

\section{Summary}

Identifying the source of excess androgens is often difficult requiring correct interpretation of symptoms, signs, biochemistry, dynamic testing and imaging.

In some cases MRI pelvis may offer improved resolution compared to US pelvis and should be considered in difficult cases.

\section{Key references}

Dolinko AV, Ginsburg ES. Hyperandrogenism in menopause: a case report andliterature review. Fertil Res Pract. 2015 May 19:1:7. doi: 10.1186/2054-7099-1-7.eCollection 2015

Alali I, Haj Hassan L, Mardini G, Hijazi N, Hadid L, Kabalan Y. Diagnostic Dilemma in Two Cases of Hyperandrogenism. Case Rep Endocrinol. 2018 Jun 27;2018:9041018. doi: 10.1155/2018/9041018. eCollection 2018 .

Braithwaite SS, Erkman-Balis B, Avila TD. Postmenopausal virilization due to ovarian stromal hyperthecosis. Braithwaite SS, Erkman-Balis B, Avila
Clin Endocrinol Metab 1978; 46:295.

Rothman MS, Wierman ME. How should postmenopausal androgen excess be evaluated? Clin Endocrino (Oxf). 2011;75:160-4.

Goldman JM, Kapadia LJ. Virilization in a postmenopausal woman due to ovarian stromal hyperthecosis. Postgrad Med J 1991; 67:304

6. Nagamani M, Hannigan EV, Dinh TV, Stuart CA. Hyperinsulinemia and stromal luteinization of the ovaries in postmenopausal women with endometrial cancer. J Clin Endocrinol Metab 1988; 67:144. Barth JH, Jenkins M, Belchetz PE. Ovarian hyperthecosis, diabetes and hirsuties in post-menopausal women. Clin Endocrinol (Oxf) 1997; 46:123.

8. DiSaia PJ, Creasman WT. Germ cell, stromal and other ovarian tumors. In: Clinical Gynecologic Oncology, Mosby-Yearbook, 1997. p.351.

9. Krug E, Berga SL. Postmenopausal hyperthecosis: functional dysregulation of androgenesis in climacteric ovary. Obstet Gynecol. 2002;99:893.

0. Agorastos T, Argyriadis N, Fraggidis G, Vakiani A, Zournatzi V, Bontis J.Postmenopausal virilization due to ovarian hyperthecosis. Arch Gynecol Obstet.1995;256(4):209-11. 